\title{
A Disorder Easy to be Misdiagnosed-benign Metastasizing Uterine Leiomyoma with Multiple Metastasis to Lungs and Pelvic Lymph Nodes Simultaneously and Literature Review
}

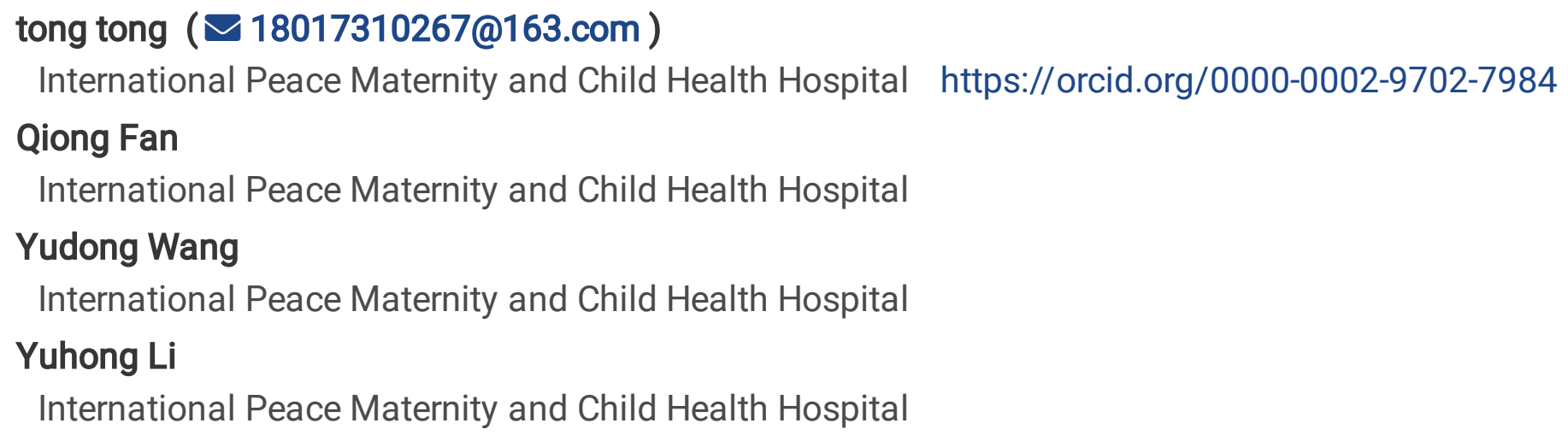

\section{Case Report}

Keywords: Benign metastasizing leiomyoma, lymph node, pulmonary metastases

Posted Date: April 13th, 2021

DOl: https://doi.org/10.21203/rs.3.rs-414297/v1

License: (c) (1) This work is licensed under a Creative Commons Attribution 4.0 International License. Read Full License 


\section{Abstract}

Background: Benign metastasizing leiomyoma $(B M L)$ is a rare disease that is usually seen in females of reproductive or premenopausal age with a previous surgery history of uterine myomectomy or hysterectomy. The most common sites of metastases are pulmonary metastases, and other sites include heart, bones, liver, lymph nodes, skin, bladder, esophagus, skeletal muscles, and central nervous system. Here we report a case of 50-year-old women with history of hysterectomy whom was first suspect uterine sarcoma but finally confirmed BML with lung and lymph node metastases at the same time and discuss the treatment and prognosis of BML.

Case presentation: A 50-year-old women who have a history of abdominal total hysterectomy presented with mild but persistent abdominal pain for over 3 months. She was suspected uterine sarcoma before surgery and laparoscopic extensive debulking surgery including bilateral oophorectomy, pelvic and paraaortic lymph node dissection to the level of the left renal vein, and right inguinal lymph nodes dissection trans-cutaneously were done. Pathology after surgery confirmed benign leiomyoma and she was diagnosed BML. No medicine was given after the surgery and now the follow up is of no significance.

Conclusions: Benign metastasizing leiomyoma $(\mathrm{BML})$ is a rare disorder in which histologically benign smooth muscle tumor metastasize to extra-uterine sites. Metastases are commonly seen in the lung, liver, lymph nodes, skin, bladder, esophagus, skeletal muscles, et al. BML is usually misdiagnosed as malignant tumor before surgery until pathology confirmed the benign nature. The treatment is controversial and undetermined. The prognosis is usually favorable because of its benign nature.

\section{Background}

Benign metastasizing leiomyoma $(\mathrm{BML})$ is a rare disorder which mainly occurs in females of reproductive or premenopausal age with a previous surgery history of uterine myomectomy or hysterectomy. Histologically, BML are benign tumors that originate from smooth muscle cells but which would metastasize to extra-uterine sites. The most common sites of metastases are pulmonary metastases, and other sites include heart, bones, liver, lymph nodes, skin, bladder, esophagus, skeletal muscles, and central

nervous system ${ }^{1-11}$. Multiple metastases at the same time including lymph nodes metastases in BML are extremely rare and studies about prognosis and treatment of BML with multiple lymph node metastasis are few. Here we report a case of BML with pulmonary and lymph nodes metastases at the same time and discuss the clinical and radiologic features of BML.

\section{Case Presentation}

A 50-year-old women with no significant past medical history (parity 2-0-0-2, both normal spontaneous vaginal deliveries) presented with mild but persistent abdominal pain for over 3 months. She had received an abdominal total hysterectomy and bi-salpingectomy for uterine leiomyoma at a local secondary hospital in January 2019. On pathology, it was found to be benign leiomyoma. The post-operative 
regular follow up was of no significance. However, she felt persistent lower abdominal pain in the recent 3 months, but did not suffer from loss of weight, or any other respiratory symptoms like hemoptysis, purulent sputum or exertional dyspnea. Ultrasound of a local clinic found a heterogeneous solid mass of $4 \mathrm{~cm}$ in diameter in the central pelvic. She was then referred to International Peace Maternity and Child Health Hospital for further work up.

Routine laboratory tests showed that the values for biochemical variables, including tumor markers such as alpha-fetoprotein, cancer antigen 19-9, and cancer antigen 125 , were within the normal ranges. Pelvic magnetic resonance imaging (MRI) revealed solid pelvic masses in the central and bi-lateral pelvis, and there were enlargement of multiple lymph nodes around the iliac chain and aorta. On chest CT, there were multiple well-circumscribed nodules in the bilateral pulmonary, with the largest nodule $1.5 \mathrm{~cm}$ in diameter (figure1). An original pathology slide review and positron emission tomography (PET)-CT were done. According to the original slide review, it was a benign leiomyoma with a mitotic count $<5 / 10$ high-power fields (HPF), little cytological atypia and no tumor cell necrosis, but with cellular abundance. Abnormal fluorodeoxyglucose (FDG) uptakes in bi-adnexal mass with the suspicion of para-aortic, aorto-caval, precaval, external iliac and bi-lateral inguinal lymph node metastases were found on PET-CT (figure 2). Multiple nodules in bi-lateral lungs without abnormal FDG uptake were considered metastatic cancer. An additional chest CT also revealed it to be a metastatic lung cancer. Considering the history of gynecologic surgery, pelvic malignancy of unknown origin was suspected. In December 2020 laparoscopic extensive debulking surgery including bilateral oophorectomy, pelvic and para-aortic lymph node dissection to the level of the left renal vein, and right inguinal lymph nodes dissection trans-cutaneously were done. Grossly, there were masses $5 \mathrm{~cm}$ in size on the left side stump of the vagina and also along the right pelvic infundibular ligament to the level of renal vein, measuring $10 \mathrm{~cm} * 4 \mathrm{~cm}$ (figure 3-4). All the lymph nodes stated above including para-aortic, aorto-caval, precaval and pelvic lymph nodes were enlarged to about $0.5 \mathrm{~cm}$ to $3 \mathrm{~cm}$ in size. Right inguinal lymph nodes were palpable and enlarged to $3 \mathrm{~cm}$ in size and were dissected.

Pathology for the pelvic masses were also benign leiomyoma with mitosis $<5 / 10 \mathrm{HPF}$, little cytological atypia and no tumor necrosis, but with cellular abundance and a few singular kernel (figure 5). Metastases to meso-ovarium, bilateral pelvic infundibular ligaments, pelvic lymph nodes, para-aortic lymph nodes, and bilateral inguinal lymph nodes were confirmed on the final pathology report. Metastatic lesions were also reported to have similar histology with the original mass with a mitotic count $<2 / 10$ HPF, little cytological atypism and no necrosis. On immunohistochemical staining, cells were all positive for estrogen/progesterone receptors, p53 positive, SMA+, HMB45-, Ki67(5\%), CD31 and ERG positive. Clinico-pathologically, BML was selected as a final diagnosis with discussion with our pathologists. The patient was discharged on post-operative day 7 from the hospital without any early post-operative complications. No specific treatment was given. Her current general condition is satisfactory without any postoperative complications. However, long-term follow-up is required for assessment of disease recurrence and distant metastases. 


\section{Discussion And Conclusions}

Benign metastasizing leiomyoma (BML) is a rare disorder in which histologically benign smooth muscle tumor metastasize to extra-uterine sites ${ }^{12}$. In 1939 Steiner $^{13}$ first reported it as fibro-leiomyomatous hamartoma in the literature.

The disease commonly occurs in women of late reproductive or premenopausal age, most of whom have a history of leiomyomas with surgical treatment with myomectomy or hysterectomy ${ }^{14}$. The most frequent site of metastasis is in the pulmonary (80\%). Metastases to other organs reported until now include the heart, liver, lymph nodes, skeletal muscle, skin, esophagus, and central nervous system. Multiple metastases are extremely rare, as reported in this case.

The pathological origin of BML remains undetermined and controversial. Several hypotheses are proposed as follows: (i) mechanical dissemination or intravascular metastases of smooth muscle cells from the uterus to distant locations, (ii) derivation from a multifocal but independent smooth muscle proliferation, and (iii) derivation from a low-grade leiomyosarcoma ${ }^{15-16}$. The most commonly accepted theory is the haematogenous spread of a monoclonal element of the benign smooth muscle tumor. In $25 \%$ of such tumors chromosomal abnormalities could be found, including balanced translocation, trisomy 12 or rearrangement of $6 \mathrm{p}^{17-18}$. Other possible mechanisms include lympho-vascular embolization, mesothelial mesenchymal metaplasia, and metastases from misdiagnosed low-grade uterine leiomyosarcomas ${ }^{19-20}$.

Histologically BML has been mainly associated with leiomyomas, however, the type of leiomyomas is not yet clear (eg. cellular, hemorrhagic cellular, mitotically active, atypical, myxoid, vascular, epithelioid, or lipoleiomyomas). Some suggest BML as a low-grade endometrial stromal sarcoma or malignant uterine leiomyoma, but the evidence supporting these concepts is not very strong.

On imaging examination, such as CT scans of the lungs, BML presents as well-circumscribed nodules in the lungs. Bilateral lung nodules are more commonly seen than multiple unilateral lesions or solitary nodule. The nodules of BML can be large or small, and cavitate, which would lead to thin-walled or thickwalled cysts ${ }^{21}$.

For PET-CT scan, some propose that 18-FDG-PET/CT is useful for distinguishing malignant leiomyosarcoma from benign leiomyoma as a number of reports have demonstrated that there is a lack of 18-FDG uptake in PBML ${ }^{22}$. Basically, SUV values of leiomyosarcoma were observed to be significantly greater than those for leiomyoma. However, there are also numerous reports for 18-FDG-avid leiomyoma. PET-CT with $18 \mathrm{~F}-\mathrm{FDG}$ in theory is helpful to assess for significant metabolic activity of both pulmonary and extra-pulmonary nodules, which should be unexpected in BML and otherwise would raise concern for malignant disease $\mathrm{e}^{23-24}$. As in our case, the PET-CT of the patient showed 18-FDG-avid in lymph nodes metastases, while lack of 18-FDG uptake in pulmonary lesions, which should raise the concern for the diagnose of BML. 
The immune-histochemical profile of BML is positive staining for actin, desmin, and smooth muscle actin and a low Ki-67 expression $(<5 \%)^{25}$, and is hard to be distinguished with primary uterine leiomyoma. High levels of the tumor suppressor gene p53 are also been found in BML lesions, but its role in pathogenesis has not been clarified.

In theory, BML are estrogen and progesterone receptor positive, and estrogen is known to stimulate while progesterone known to inhibit tumor growth ${ }^{26}$. However, in a few cases, BML cells are found to be in lacked of estrogen or progesterone receptors, which suggest that the site of origin was not the uterus. Another possible explanation is that the receptors had been down-regulated. There are genetic studies that report that $B M L$ is clonal in origin. Most of the BML lesions have been reported to be nonreactive to the antibody HMB-45, which recognize Pmel17, a melanosomal protein expressed in lung hamartomas, PEComas, and LAM, however, the BML lesions may also show low reactivity.

Most of the BML patients present with multiple pulmonary nodules which usually develop some time after a myomectomy or hysterectomy for treatment of uterine fibroids. Until now, there is no standard clinical criteria for the diagnosis of BML. For many cases, lung metastases of BML are usually asymptomatic. Lung biopsies have been used to confirm a diagnosis of BML and rule out malignant tumors. For lymph nodes metastases of BML, as in our case, leiomyomatosis must be distinguished from several other spindle cell tumors involving the lymph nodes (primarily or secondary) such as Kaposi's sarcoma, dendritic reticulum cell tumour, intranodal neurilemmoma and metastatic spindle cell tumour.

To rule out many of the listed disorders, a careful history and physical examination is useful. Lack of systemic symptoms or no symptoms at all, along with a history of uterine fibroids or prior uterine surgery is highly suggestive of BML. CT imaging of the chest and abdomen may suggest malignancy tumors or infection. Ultimately, histologic diagnosis would be required to rule out the diagnoses listed above and establish the diagnosis of BML. This may consist of a lung biopsy and lesion resection.

Given the rarity of the disease, no standardized treatment for BML has been proposed. Several suggestions published in the literature include regular and careful observation, surgical resection with oophorectomy, progesterone therapy, and medical castration using aromatase inhibitors, oestrogen receptor blockers (e.g. tamoxifen, raloxifen), tyrosine kinase inhibitors (e.g.imatinib) and GnRH agonists ${ }^{27-29}$. Jennifer $B^{12}$. et al. reported a case of pelvic leiomyoma with lung metastases who underwent bi-salpingo-oophorectomy and opted for HRT after the surgery. Thoratic CT scan showed enlarged pulmonary nodule and stopped HRT. Letrozole was given by gynecologic oncologist for slightly enlarged lung nodule on CT surveillance. Two years later, surveillance imaging has confirmed stability of her pulmonary BML and the patient remains asymptomatic. This implicated the estrogen impact on the tumor and HRT treatment should be carefully assessed before given to the patient. Careful follow up is also indicated. In our case, the patient had bi-oophorectomy and no special treatment was given. The follow up until now is uneventful. 
The reported prognosis of patients with BML is favorable. The pulmonary lesions are usually discovered many years (months to more than 30 years) after hysterectomy or myomectomy and the growth rate of these tumors is slow. Although lung BML is rare and the simultaneous metastases of the retroperitoneal lymph nodes after previous hysterectomy or myomectomy is even rarer, such patients are suggested to have prolonged surveillance and careful follow-up for early detection of recurrence or distant metastases. As the therapeutic options are limited, new drugs or new therapeutic strategies should be studied and considered. In addition, long-term follow-up may be useful for the study of their implication in clinical practice.

\section{Abbreviations}

BML: benign metastasizing leiomyoma

MRI: magnetic resonance imaging

PET: positron emission tomography

HPF: high-power fields

FDG: fluorodeoxyglucose

SUV: standard uptake value

\section{Declarations}

\section{- Ethics approval and consent to participate}

The institutional review board (International Peace Maternity and Child Health Hospital) approved this work

\section{- Consent for publication}

Informed consent for publication of clinical data/details/images was obtained from patient. A copy of consent is available for review by the Editor of this journal

\section{- Availability of data and materials}

There is no dataset as this is a case report. Data/details of the patient available upon request

\section{- Competing interests}

The authors declare that they have no competing interests 


\section{- Funding}

This research did not receive any specific grants or funding

\section{- Authors' contributions}

TT: writing of the manuscript. FQ: providing the case details. LYH: writing and editing of the manuscript. WYD: editing of the manuscript. The authors read and approved the final manuscript

\section{- Acknowledgements}

Not applicable.

\section{References}

1. Jo JH, Lee JH, Kim DC, et al. A case of benign metastasizing leiomyoma with multiple metastasis to the soft tissue, skeletal muscle, lung and breast. Korean J Intern Med 2006;21(3):199-201.

2. Arai T, Yasuda Y, Takaya T, et al. Natural decrease of benign metastasizing leiomyoma. Chest 2000;117(3):921-2.

3. Kwon Yl, Kim TH, Sohn JW, et al. (2006) Benign pulmonary metastasizing leiomvomatosis: case report and a review of the literature. Korean J Intern Med 21(3):173-177.

4. Rivera JA, Christopoulos S, Small D, et al. (2004) Hormonal manipulation of benign metastasizing leiomyomas: report of two cases and review of the literature. J Clin Endocrinol Metab11:3183-3188.

5. G. Jautzke, E. Müller-Ruchholtz, U. Thalmann, Immunohistological detection of estrogen and progesterone receptors in multiple and well differentiated leiomyomatous lung tumors in women with uterine leiomyomas (so-called benign metastasizing leiomyomas): a report on 5 cases, Pathol. Res. Pract. 192 (3) (1996 Jan 1) 215-223.

6. Goyle KK, Moore DF Jr, Garrett C, et al. (2003) Benign metastasizing leiomyomatosis: case report and review. Am J Clin Oncol 11:473-476.

7. Schneider T, Kugler C, Kayser K, et al. (2001) Benign uterine leiomyoma with metastatic pulmonary nodales. Chirurg72(3):308-311.

8. Andrade L A, Torresan Z Renato, Sales FS Jose, et al. Intravenous leiomyomatosis of the uterus. A report of three cases, Pathol Oncol Res: POR 4:44-47, 1998.

9. Abramson S, Gilkeson RC, Goldstein JD, et al. (2001) Benign metastasizing leiomyoma: clinical, imaging, and pathologic correlation. AJR Am J Roentgenol 176(6):1409-1413.

10. Yoon G, Kim TJ, Sung CO, et al.: Benign metastasizing leiomyoma with multiple lymph node metastasis: A case report. Cancer Res Treat 43: 131-133, 2011.

11. Egberts JH, Schafmayer C, Bauerschlag DO, et al. Benign abdominal and pulmonary metastasizing leiomyoma of the uterus. Arch Gynecol Obstet 274:319-322, 2006. 
12. Jennifer Bakkensen, Wesley Samore, Pietro Bortoletto, Pelvic and pulmonary benign metastasizing leiomyoma: A case report, Case Reports in Women's Health 18 (2018) e00061.

13. Steiner PE (1939) Metastasizing fibro-leiomyoma of the uterus: report of a case and review of the literature. Am J Pathol 15:89-110.7.

14. E. Barnaś, M.Książek, R. Raś, A. Skręt, J. Skręt-Magierło, E. Dmoch-Gajzlerska, Benign metastasizing leiomyoma: a review of current literature in respect to the time and type of previous gynecological surgery, PLoS One 12 (4) (2017 Apr 20), e0175875.

15. H.J. Lee, J. Choi, K.R. Kim, Pulmonary benign metastasizing leiomyoma associated with intravenous leiomyomatosis of the uterus: clinical behavior and genomic changes supporting a transportation theory, Int. J. Gynecol. Pathol. 27 (3) (2008 Jul 1) 340-345;

16. R.C. Wu, A.S. Chao, L.Y. Lee, et al., Massively parallel sequencing and genome-wide copy number analysis revealed a clonal relationship in benign metastasizing leiomyoma, Oncotarget 8 (29) (2017) 47547-47554.

17. Esteban JM, Allen WM, Schaerf RH (1999) Benign metastasizing leiomyoma of the uterus: histologic and immunohistochemical characterization of primary and metastatic lesions. Arch Pathol Lab Med 123:960-962.

18. Uchida T, Tokumaru T, Kojima H, Nakagawaji K, Imaizumi M, Abe T (1992) Acase of multiple leiomyomatous lesions of the lung: an analysis of flow cytometry and hormone receptors. Surg Today 22:265-268.

19. Beck MM, Biswas B, D'Souza A, Kumar R (2012) Benign metastasising leiomyoma after hysterectomy and bilateral salpingo-oophorectomy. Hong Kong Med J 18:153-155.

20. Awonuga AO, Rotas M, Imudia AN, Choi C, Khulpateea N (2008)Recurrent benign metastasizing leiomyoma after hysterectomy and bilateral salpingo-oophorectomy. Arch Gynecol Obstet 278:373376.

21. Gustavo Pacheco-Rodriguez, Benign Metastasizing Leiomyoma, Clin Chest Med 37 (2016) 589-595.

22. Yusuke S., Toshiki S., Yuta Y. Benign metastasizing leiomyoma and 18-FDG-PET/CT: A case report and literature review. ONCOLOGY LETTERS 14: 3641-3646, 2017.

23. S. Chen, R.M. Liu, T. Li, Pulmonary benign metastasizing leiomyoma: a case report and literature review, J. Thorac. Dis. 6 (6) (2014 Jun), E92.

24. Feras Abu Saadeh, Ciaran Riain, Noreen Gleeson. Lung metastases from benign uterine leiomyoma: does 18-FDG-PET/CT have a role to play? Irish Journal of Medical Science (1971 -) (2019) 188:619624;

25. K. Kayser, S. Zink, T. Schneider, et al., Benign metastasizing leiomyoma of the uterus: documentation ofclinical, immunohistochemical and lectin-histochemical data of ten cases, Virchows Arch. 437 (3) (2000 Sep 7) 284-292.

26. G. Jautzke, E. Müller-Ruchholtz, U. Thalmann, Immunohistological detection of estrogen and progesterone receptors in multiple and well differentiated leiomyomatous lung tumors in women 
with uterine leiomyomas (so-called benign metastasizing leiomyomas): a report on 5 cases, Pathol. Res. Pract. 192 (3) (1996 Jan 1) 215-223.

27. A.S. Banner, C.B. Carrington, W.B. Emory, et al., Efficacy of oophorectomy in lymphangioleiomyomatosis and benign metastasizing leiomyoma, Obstet. Gynecol. Surv. 36 (11) (1981 Nov 1)653-655

28. W.M. Hague, N.A. Abdulwahid, H.S. Jacobs, I. Craft, Use of LHRH analogue to obtain reversible castration in a patient with benign metastasizing leiomyoma, BJOG Int.J. Obstet. Gynaecol. 93 (5) (1986 May 1) 455-460

29. K. Nasu, A. Tsuno, N. Takai, H. Narahara, A case of benign metastasizing leiomyoma treated by surgical castration followed by an aromatase inhibitor, anastrozole, Arch. Gynecol. Obstet. 279 (2) (2009 Feb 1) 255-257.

\section{Figures}

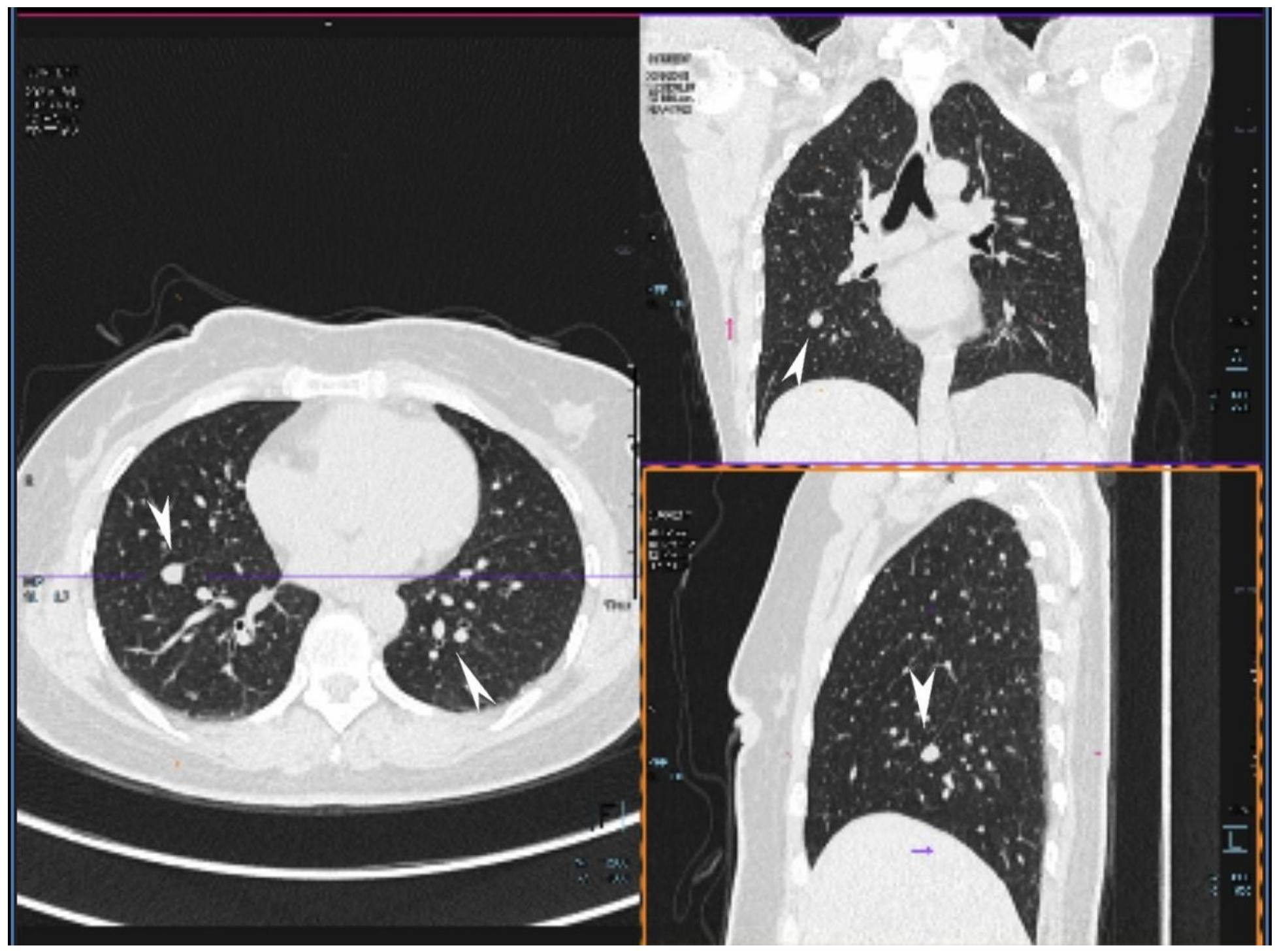

Figure 1 


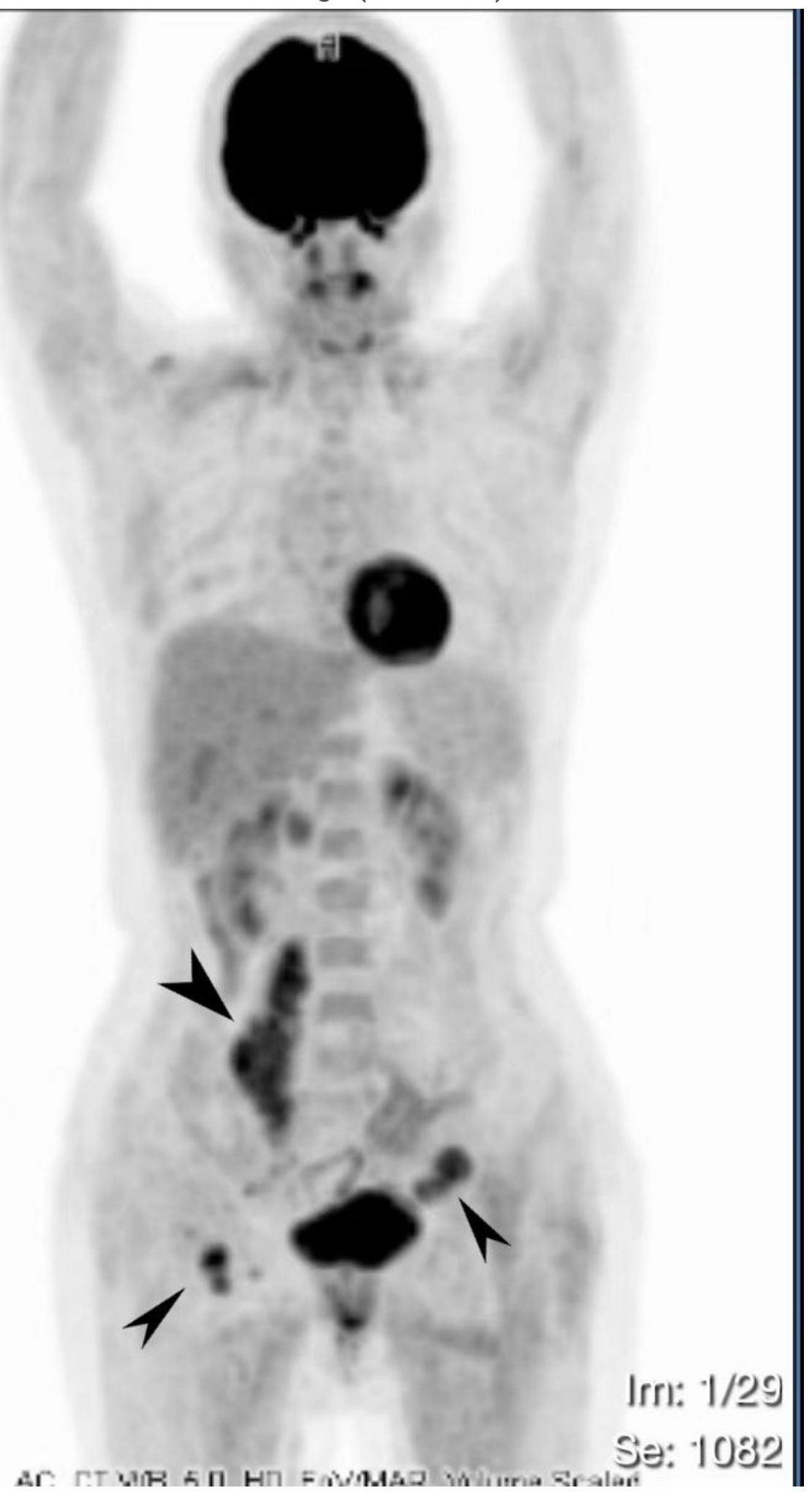

Figure 2

Multiple metastasis in pelvic, along post cava and the right inguinal lymph nodes. 


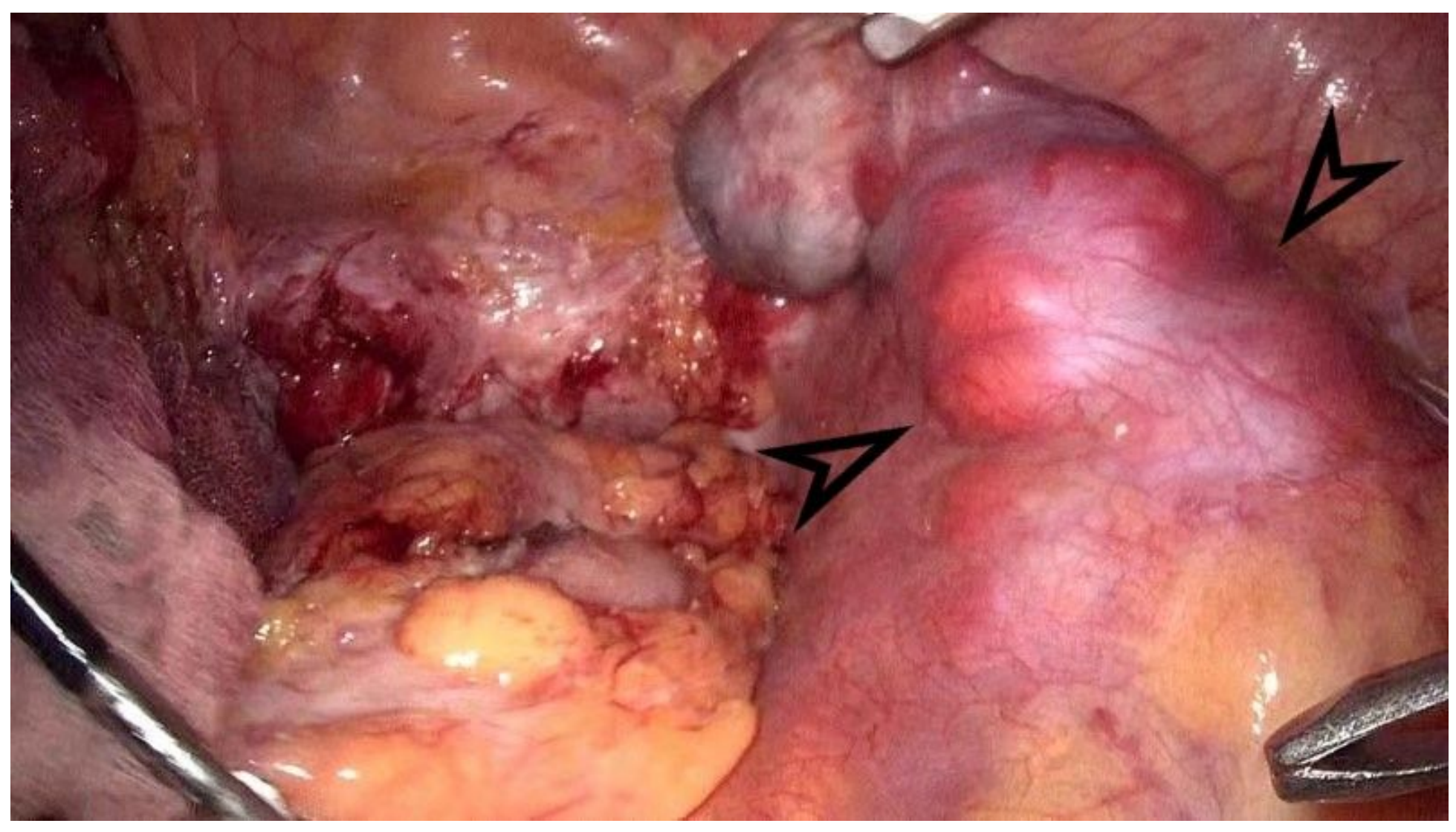

Figure 3

leiomyoma metastasis along the post cava seen in the laparoscopic surgery.

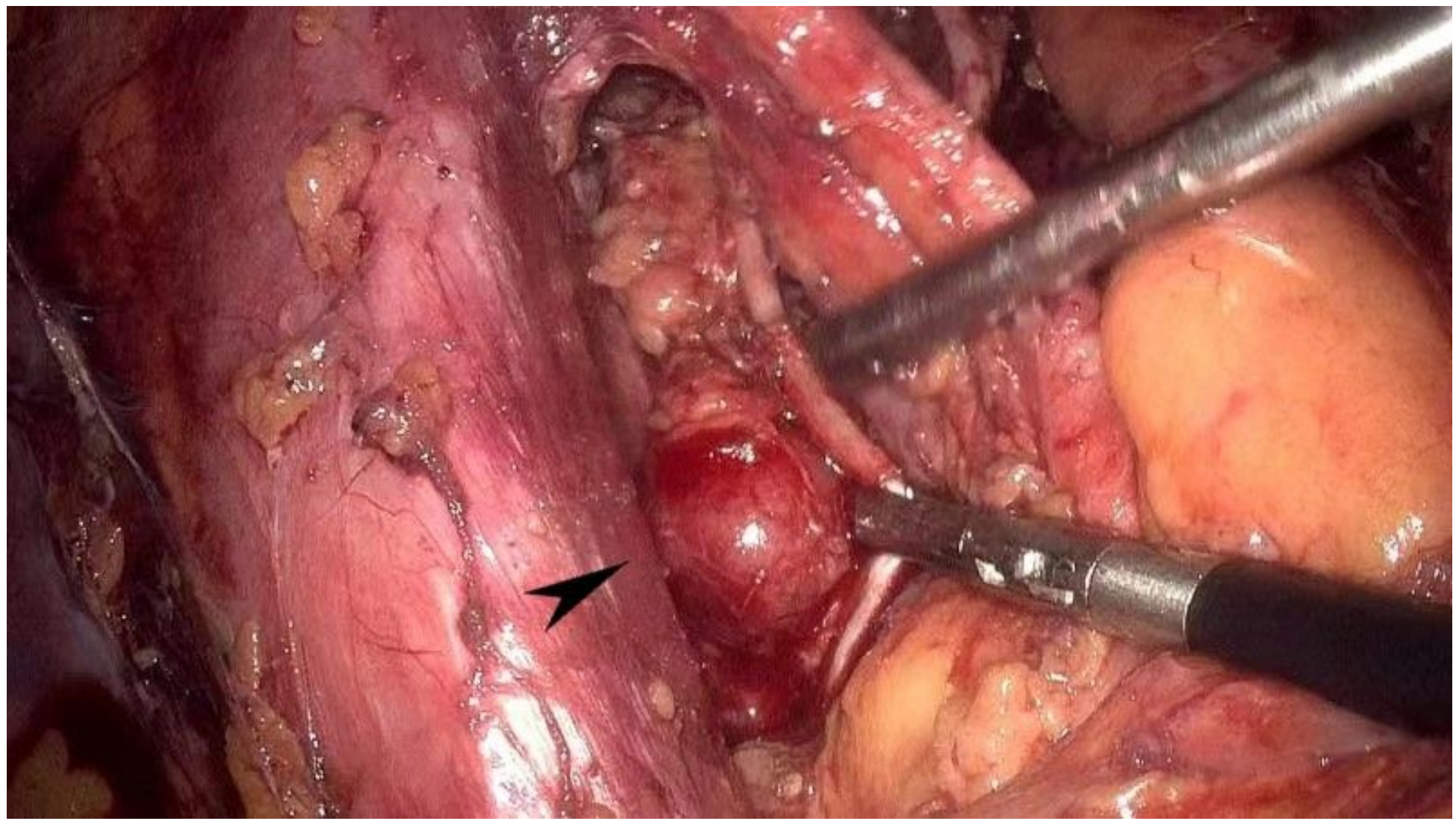


Figure 4

leiomyoma metastasis to the internal iliac lymph nodes seen in the laparoscopic surgery.

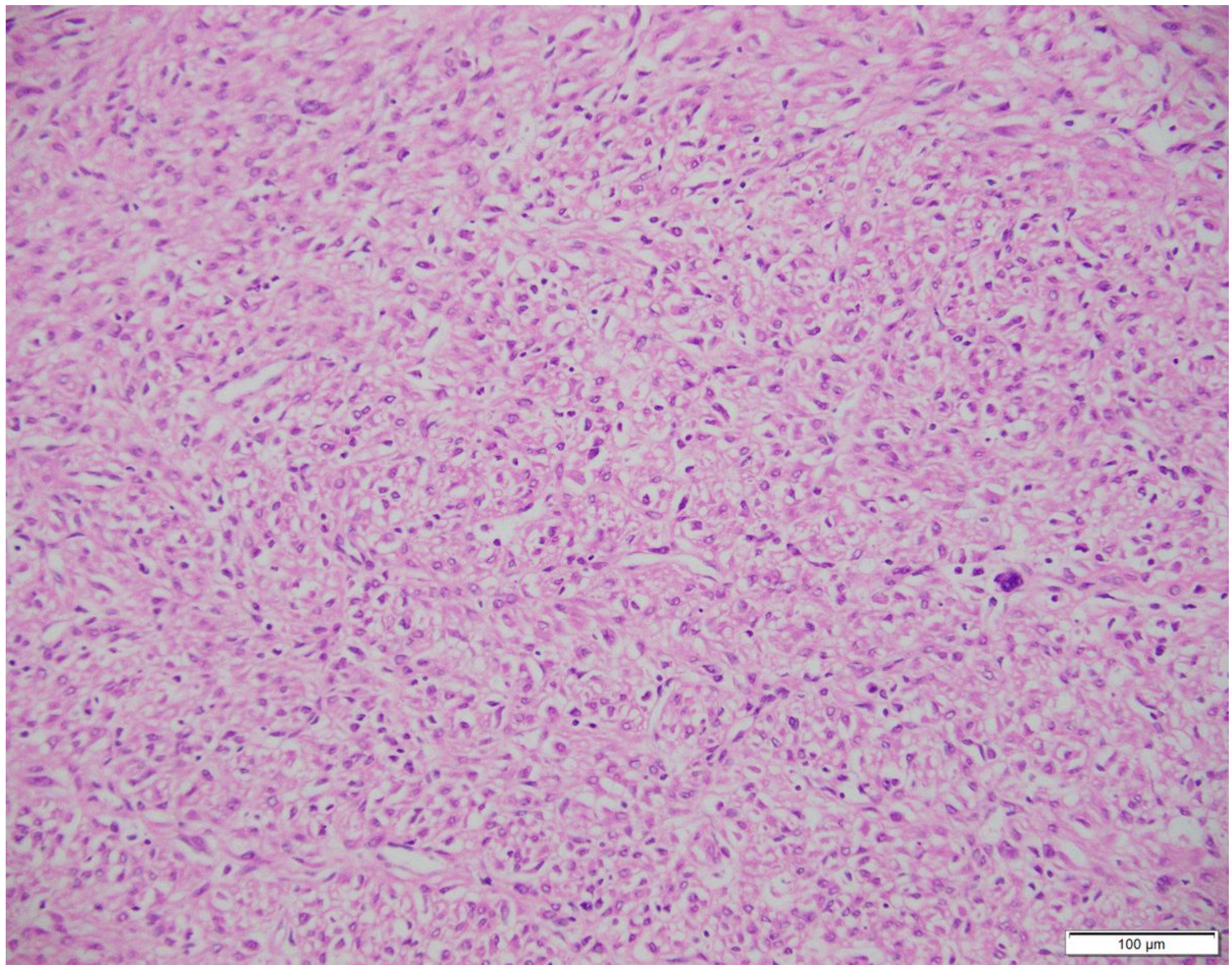

Figure 5

Hematoxylin and eosin(HE) staining of the pelvic tumor specimen

\section{Supplementary Files}

This is a list of supplementary files associated with this preprint. Click to download.

- CAREchecklistEnglish.pdf 